University of Massachusetts Amherst

ScholarWorks@UMass Amherst

1996

\title{
Flow Injection Determination of Mercury with Preconcentration by Amalgamation on a Gold- Platinum Gauze by Inductively Coupled Plasma Mass Spectrometry
}

Ebenezer Debrah

Eric R. Denoyer

Julian Tyson

University of Massachusetts Amherst

Follow this and additional works at: https://scholarworks.umass.edu/chem_faculty_pubs

Part of the Chemistry Commons

\section{Recommended Citation}

Debrah, Ebenezer; Denoyer, Eric R.; and Tyson, Julian, "Flow Injection Determination of Mercury with Preconcentration by Amalgamation on a Gold-Platinum Gauze by Inductively Coupled Plasma Mass Spectrometry" (1996). Journal of Analytical Atomic Spectrometry. 1393.

Retrieved from https://scholarworks.umass.edu/chem_faculty_pubs/1393 


\title{
Flow Injection Determination of Mercury With Preconcentration by Amalgamation on a Gold-Platinum Gauze by Inductively Coupled Plasma Mass Spectrometry
}

\author{
EBENEZER DEBRAH AND ERIC R. DENOYER \\ The Perkin-Elmer Corporation, 50 Danbury Road, Wilton, CT 06897-0215, USA \\ JULIAN F. TYSON \\ Chemistry Department, University of Massachusetts, Amherst, MA 01003, USA
}

Trace concentrations of mercury in water samples were determined by a method involving a preconcentration procedure and inductively coupled plasma mass spectrometry (ICP-MS) detection. Mercury vapour, generated by tin(II) chloride as the reductant, was trapped by amalgamation on a gold-platinum gauze, released by controlled heating and detected by ICP-MS. A flow injection sample introduction system with time-based injection was used and the sensitivity was found to be proportional to the mass of mercury introduced. Quantitative recoveries of mercury from open ocean sea-water, coastal sea-water and fresh water reference materials were obtained. A detection limit of $200 \mathrm{pg} \mathrm{l}^{-1}$ for a $25 \mathrm{ml}$ sample was obtained. The precision of the method was $1 \%$ (relative standard deviation) at $1 \mu \mathrm{g} \mathrm{I}^{-1}$.

Keywords: Preconcentration; amalgamation; flow injection; inductively coupled plasma mass spectrometry

The determination of the chemical composition of soil, water, air and living organisms provides a basis for the study of natural and man-made influences on the environment. Trace elements play an important role in establishing the processes affecting these interactions. As the results of long-term detailed studies become available, there is a growing need for reliable measurements of lower and lower concentrations. ${ }^{1}$ The high toxicity of mercury and some of its compounds makes the determination of very low concentrations of this element in a variety of samples, especially water, very important.

The high sensitivity and freedom from spectral interferences exhibited for most elements determined by inductively coupled plasma mass spectrometry (ICP-MS), compared with other atomic spectrometric techniques, makes it a very attractive choice for trace element analysis. Although most elements in the Periodic Table are ionized with an efficiency of more than $90 \%$ in the argon plasma, mercury, with an ionization potential of $10.437 \mathrm{eV}$, is ionized with only $32.31 \%$ efficiency. ${ }^{2,3}$ This results in a decrease in detection power for mercury. In samples containing very low concentrations or samples that have to be diluted in order to diminish matrix effects, detection capabilities become restricted, making analysis difficult or impossible. Preconcentration procedures improve sensitivity, and usually the detection limit achievable. Flow injection (FI) sample preconcentration methods reduce the risk of contamination since FI systems are closed. This is especially important for determinations of $\mathrm{ng}^{-1}$ concentrations where high blank signals could lead to a deterioration in precision of the signal-tobackground ratio and a degradation in detection limit.

Vapour generation techniques have been used extensively in the sensitive determination of mercury by atomic absorption spectrometric (AAS) detection..$^{4-9}$ A variant of this procedure, for multi-element determinations, has been the use of procedures that allow other vapour-forming analytes to be gener- ated. ${ }^{10}$ In this way several analytes of interest may be determined simultaneously with a multi-element detection system such as inductively coupled plasma optical emission spectrometry or mass spectrometry (ICP-OES or ICP-MS). An FI method for the preconcentration and determination of mercury in biological materials using ICP-OES detection has been described by Rudner et al. ${ }^{11}$ In this method, a complex of mercury was formed by reaction with 1,5-bis(di-2-pyridyl)methylene thiocarbonohydrazide, which was then extracted into isobutyl methyl ketone. The organic extract was mixed on-line with tin(II) chloride in dimethylformamide to generate the cold vapour of mercury for detection and quantification. A detection limit of $2 \mu \mathrm{g} 1^{-1}$ was obtained. Stroh and Vollkopf ${ }^{12}$ have reported procedures for the ICP-MS determination of $\mathrm{As}, \mathrm{Sb}$ and $\mathrm{Hg}$ in standard reference sea-waters in which sodium tetrahydroborate was used as reductant. Using sodium tetrahydroborate as the reducing agent and ICP-MS detection, Haraldsson et al. ${ }^{13}$ have reported on the determination of mercury in natural waters and reference sediments. A detection limit of $8 \mathrm{pg}$ based on a total blank volume of $100 \mathrm{ml}$ was claimed. An on-line flow system for the elimination of interferents from alkali and alkaline earth elements in the determination of mercury and other elements in sea-water by ICP-MS has been proposed by Plantz et al. ${ }^{14}$ Bis(carboxymethyl)dithiocarbamate was used to form complexes with mercury and the other elements which were retained under acidic conditions, on a column packed with an Amberlite polymeric adsorbent, XAD-4, while interferents passed freely through. The elements were eluted with aqueous ammonia. A detection limit for mercury of $0.04 \mu \mathrm{g} \mathrm{l}^{-1}$ was obtained with this procedure. Isotope dilution ICP-MS has been used for the determination of mercury in biological SRMs. ${ }^{15}$ In this method, two marine biological reference materials, dogfish liver tissue and dogfish muscle tissue, were digested in a nitric acid-hydrogen peroxide mixture for the accurate determination of mercury and other trace elements by ICP-MS.

The objective of the present work was to develop a simple method for the accurate ICP-MS determination of mercury in aqueous samples at concentrations as low as $1 \mathrm{ng}^{-1}$. The method is based on flow injection sample introduction, the generation of mercury vapour with tin(II) chloride and preconcentration by amalgamation. The method separates potentially interfering matrix components from the analyte.

\section{EXPERIMENTAL}

Instrumentation

ICP-MS

An ELAN 5000 (Perkin-Elmer SCIEX, Thornhill, Ontario, Canada) ICP-MS instrument was used for all the analyses. 
Table 1 Optimized operating conditions used for the ELAN 5000 ICP-MS instrument

Forward if power

Plasma argon flow rate

Auxiliary argon flow rate

Carrier argon flow rate

Sampler and skimmer cones

Data acquisition

Scan mode

Points per spectral peak

For vapour introduction, the ICP torch was fitted with a standard vapour adapter. For solution introduction, to optimize the alignment of the torch a gem-tipped chemically resistant Ryton cross-flow nebulizer and a Ryton spray chamber were used. Table 1 summarizes the operating conditions.

\section{Data acquisition and processing}

The ELAN software allows data to be collected and processed in peak height or peak area mode. The software also allows data that have been collected in one processing mode to be reprocessed to give the results in another mode. For instance, data collected in peak height mode could be reprocessed to give the results in peak area mode and vice versa. In the ELAN software, data acquisition parameters are entered via the Parameter Entry Form; Table 2 shows the parameters used. The element(s) to be determined are specified and the integration time and dwell time are also entered in this form. Peak area was used to characterize analytical response in these studies, because this processing mode provided better precision.

\section{Flow injection system}

A FIAS 400 FI system with AS 90 random access autosampler (Bodenseewerk Perkin-Elmer, Überlingen, Germany) was used. The flow injection system consisted of two peristaltic pumps, an injection valve and required tubing and was fully controlled by the ELAN-FIAS spectrometer software through a personal computer (IBM PS/2 Model 70). Operating parameters, such as pump speed, injection and control of the amalgam system were entered in an FIAS program. Two types of gas-liquid separators were tested. The first gas-liquid separator, evaluated by Hanna et al., ${ }^{16}$ was a cylindrical chamber partially filled with glass beads, with a separate connection to pump spent liquid to waste. The other gas-liquid separator was made of chemically resistant plastic and contained an exchangeable PTFE membrane filter. The manifold used in this study is shown in Fig. 1.

\section{Amalgam system}

The amalgam system (Bodenseewerk Perkin-Elmer) was used to concentrate the mercury vapour. The atomic mercury vapour, which is generated by reaction with a reductant, first passes through a fibre filter where any water is removed. The trapping medium was a $1.2 \mathrm{~cm}$ length of rolled gold-platinum

Table 2 ELAN 5000 parameters set used for data acquisition

\begin{tabular}{ll}
\hline Dwell time & $50 \mathrm{~ms}$ \\
Scan mode & Peak-hop transient \\
Sweeps per reading & 2 \\
Resolution & $0.8 \mathrm{u}$ at $10 \%$ peak maximum \\
Signal processing & Spectral peak integrated; \\
& signal profile counted \\
Readings per replicate & 150 \\
Isotope measured & ${ }^{202} \mathrm{Hg}$ \\
Number of replicates & 3 \\
\hline
\end{tabular}

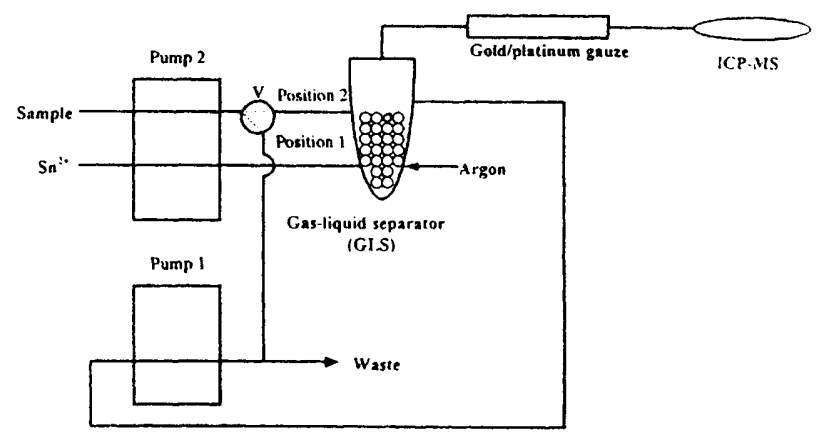

Fig. 1 Flow injection manifold for amalgamation-ICP-MS. The sample is introduced via the valve, $\mathrm{V}$, and mixed with reductant and introduced into the gas-liquid separator (GLS)

gauze inserted into a quartz tube $(0.3 \mathrm{~cm}$ id $)$. The argon supply to the manifold was controlled by the mass flow system of the ELAN 5000 instrument. The gauze was heated by two $10 \mathrm{~W}$ tungsten filament lamps. The system was cooled by compressed air.

\section{Chemicals}

Tin(II) chloride (5.5 g) (R\&D grade) (Johnson Matthey Electronics, Ward Mill, MA, USA) was dissolved in $15 \mathrm{ml}$ of ultrapure hydrochloric acid, purified by sub-boiling distillation in a quartz still (Seastar Chemicals, Seattle, WA, USA), and made up to $500 \mathrm{ml}$ with $18 \mathrm{M} \Omega \mathrm{cm}$, distilled, de-ionized water (Modulab Analytical Research Grade, Continental Water System Corporation, San Antonio, TX, USA) and stored in a polyethylene bottle. Standards were prepared daily by serial dilution of a $1000 \mathrm{mg} \mathrm{l}^{-1}$ mercury stock solution (PE Express, Norwalk, CT, USA). Optimization procedures were carried out with a $1 \mu \mathrm{g}^{-1}$ mercury solution. The NIST SRMs $1641 \mathrm{c}$ and $1643 \mathrm{c}$ were obtained from the National Institute of Standards and Technology (Gaithersburg, MD, USA) and the NRCC CRMs SLRS-1, CASS-2, NASS-2 and NASS-4 from the National Research Council of Canada (Ottawa, Canada).

\section{Time-based Analysis}

In time-based injection, a sample is loaded for a precise time at a given flow rate. Unlike volume-based injection, the volume of sample delivered can be varied without the need to change manifold parts. In time-based injection the volume delivered is determined by the flow rate or pump speed, the time allowed for the sample to flow, as well as the inner diameter of the pump tubing. The injected volume is calculated as the product of flow rate and loading time. The use of time-based injection eliminates the need to use a carrier in the flow injection system as required in a volume-based injection. This is considered an advantage in ICP-MS work since the greater the number of reagents used, the greater the risk of contamination. Timebased injection has the obvious advantage that sample volume may be changed at will, and hence an extended dynamic range can be achieved conveniently.

\section{Procedures}

\section{Optimization}

These general optimization procedures were carried out routinely whenever ICP-MS was used to ensure that the instrument met the manufacturer's stated specifications. The instrumental operating conditions were optimized while aspirating a solution containing $10 \mu \mathrm{g} \mathrm{I}^{-1} \mathrm{Mg}, \mathrm{Rh}$ and $\mathrm{Pb}$. The torch was aligned to the mass spectrometer sampler using an $x-y-z$ translation stage on which the torch is mounted, until 
the maximum sensitivity was obtained. The nebulizer flow rate was also adjusted to give the sensitivity specifications of the manufacturer. The levels of oxides and doubly charged ions were also optimized to meet the manufacturer's specification. Once these procedures had been carried out, the plasma was extinguished and the nebulizer spray chamber assembly was carefully removed, without disturbing the torch position, from the base of the torch and replaced with the special vapour adapter which was used in conjunction with the amalgam system. The generator interface design of the mass spectrometer in the ELAN 5000 instrument ensures fixed ion energies, ${ }^{17}$ so the optimum operating conditions are identical for a wet and a dry plasma. As a result no special optimization of the ICP-MS system was necessary for the vapour generation experiments once the optimization had been performed using solution nebulization.

\section{Description of the FIAS control programme}

The FIAS control programme used is shown in Table 3. A description of each step of the programme is given below.

The FIAS valve was used to introduce the sample from the AS 90 autosampler into the gas-liquid separator. The valve position determines the direction of sample flow (to waste or to gas-liquid separator). The function of Pump 1 is to pump the liquid waste products of the reaction between mercury and tin(II) chloride to waste. Pump 2 has two functions: (1) it transports the sample from the AS 90 autosampler to the vapour generation manifold and (2) it feeds the chemifold with reductant from the reductant reservoir.

\section{Pre-sample step}

Tubing connecting the AS 90 autosampler sample probe to the FIAS valve is rinsed and filled with sample using Pump 2. The valve is in position 1 (see Fig. 1) and is directing the sample to waste. At the same time Pump 2 is used to prime the chemifold with reductant. Pump 1 pumps material out of the gas-liquid separator to waste. This step is necessary to ensure that the tubing from the autosampler to the valve is filled with the sample before a predetermined amount is delivered into the gas-liquid manifold when the valve is switched.

\section{Step 1: Load/preconcentration step}

The valve is turned to position 2 and the sample from the AS 90 autosampler and reductant are introduced into the vapour generation flow system. The amount of sample introduced is determined by the flow rate and time set for this step. At the same time, using the remote switch closure functionality of the ELAN-FIAS software, the amalgam system is triggered to 'cool'.

\section{Step 2: Standby}

The valve is returned to position 1 to shut off the supply of sample. In this way a predetermined amount of sample is delivered. The sample that has been collected on the goldplatinum gauze is further cooled for the duration of this step.

\section{Step 3: Heat and read trigger}

The heating cycle on the amalgam system and the ELAN read cycle are triggered in this step. The lamps in the amalgam system raise the temperature of the gold-platinum gauze to approximately $600^{\circ} \mathrm{C}$, to release the mercury.

\section{Cool}

The cooling cycle of the amalgam system is triggered. Compressed air cools the quartz tube and gold-platinum gauze in the amalgam system in preparation for the next sample.

\section{Post-run step}

The Pump 2 is operated continuously (time set to '-') at a

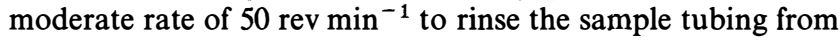
the AS 90 autosampler to the valve with a wash solution. Pump 1 is also set to run continuously to remove the wash liquid from the gas-liquid separator.

\section{Method Development}

A number of operating parameters and chemical variables of the system were studied with a view to selecting conditions to give high sensitivity and low noise. These included the concentration of the tin(II) solution, the argon gas flow rate through the gas-liquid separator, the type of gas-liquid separator, the flow rates of sample and reagent, the rf power and the sample volume. Previous experience with FI coupled with atomic spectrometry detectors suggests that many of the variables are independent and that if initial conditions are selected based on previous knowledge and expected behaviour of the system, a single cycle alternating variable search of the factor space is an adequate optimization procedure.

\section{RESULTS AND DISCUSSION}

\section{Investigation of the Effect of the Reductant Concentration}

Tin(II) chloride was used as the reducing agent in order to avoid interference effects which may occur with the more powerful sodium tetrahydroborate, in the presence of other reducible elements such as $\mathrm{Cr}, \mathrm{Fe}$ and $\mathrm{Ni}^{18}$ This was investigated by looking at the analytical response as a function of varying the concentration of tin(II) chloride. The results, presented in Fig. 2, show that there is a threshold concentration of $0.4 \%(\mathrm{~m} / \mathrm{v})$ tin(II) chloride in $3 \% \mathrm{v} / \mathrm{v} \mathrm{HCl}$ that is needed for the optimum response. Beyond this concentration, the response is not significantly affected by tin(II) chloride concentration. A tin(II) chloride concentration of $1.1 \% \mathrm{~m} / \mathrm{v}$ was therefore chosen for further experiments. This concentration is consistent with that used for cold vapour generation in the AAS determination of mercury with tin(II) chloride as the reductant. ${ }^{19}$

Table 3 FIAS programme for FI-amalgamation-ICP-MS

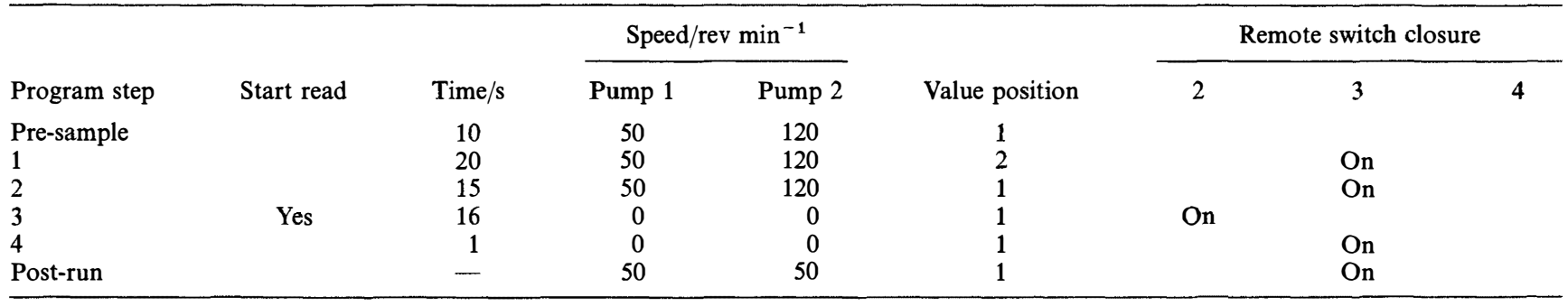




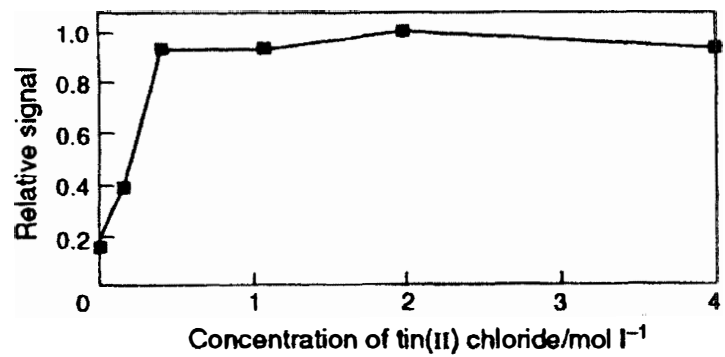

Fig. 2 Effect of the concentration of tin(II) chloride on the sensitivity of mercury

\section{Investigation of the Purge Gas Flow Rate}

For the gas-liquid separator containing beads the effect of the purge gas flow rate on the sensitivity is shown in Fig. 3. The results show that at low purge gas flow rates not all the mercury is purged out of the gas-liquid separator whereas at high purge gas flow rates the efficiency of the amalgamation process is reduced and as a result there is reduction in the analytical response in both instances. A purge gas flow rate of $11 \mathrm{~min}^{-1}$ was found to be the optimum. This purge gas flow rate is consistent with what has been reported previously. ${ }^{12}$ This study also indicates that while the kinetic effects of the gas generation reaction may be decoupled from the amalgamation process, the length of time allowed for mercury to be in contact with the gold-platinum gauze in the amalgam system as well as the amount of mercury vapour generated determines the attainable sensitivity.

The other gas-liquid separator, made of chemically resistant plastic and containing an exchangeable PTFE membrane filter to prevent liquid from being carried into the ICP-MS system, was also tested. This gas-liquid separator did not work as efficiently as the former because at the high flow rate used for the purge gas in ICP-MS, the PTFE membrane became deformed and the liquid flowed freely into the amalgam system and into the ICP torch and extinguished the plasma. In order to avoid the deformation of the PTFE membrane, the purge gas flow rate had to be reduced but this resulted in a severe loss of sensitivity. Consequently, the gas-liquid separator with glass beads was used throughout the remainder of these studies.

\section{Investigation of Memory Effects}

Memory effects were tested by measuring blanks after analysis of a $1 \mu \mathrm{g} \mathrm{l}^{-1}$ standard. Blanks were measured continuously with no rinsing between. The results, shown in Fig. 4, indicate that a rinse time of at least $50 \mathrm{~s}$, which is the equivalent time required to run one blank sample, will be sufficient to eliminate any memory effects. A rinse time of $50 \mathrm{~s}$ was used for the remainder of the experiments.

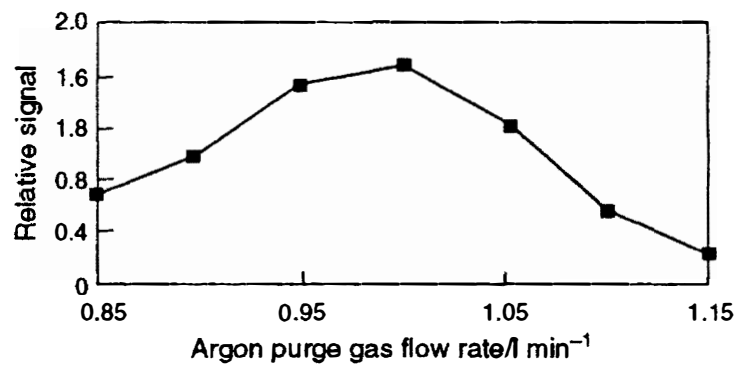

Fig. 3 Effect of flow rate of the purge gas on the analytical sensitivity of mercury

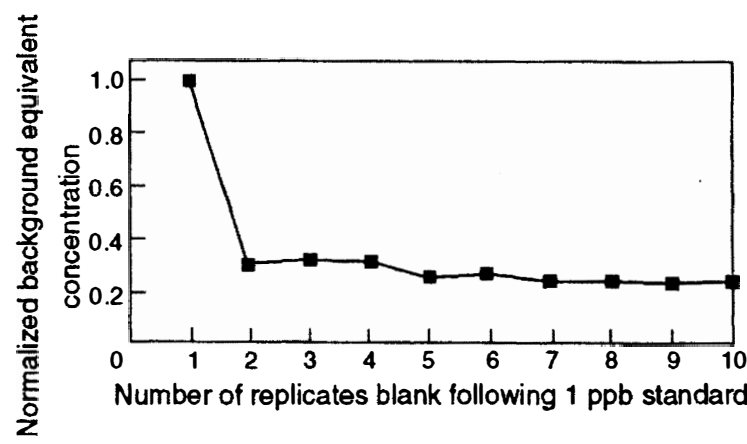

Fig. 4 Blank response following analysis of $1 \mu \mathrm{g} \mathrm{l^{-1 }}$ with no rinsing between injections

\section{Investigation of the Effect of the Flow Rate of the Sample in the Generation of the Mercury Vapour on the Analytical Response}

No significant variation in the analyte response with the rate of sample delivery into the chemifold was observed over the flow rates from 1 to $6 \mathrm{ml} \mathrm{min}{ }^{-1}$. The same amount of sample and reductant was used for the different flow rates by adjusting the times appropriately in step 1 of the FIAS programme, shown in Table 3. This study indicates that amalgamation of the mercury vapour on the gold-platinum gauze has no measurable kinetic dependence within the flow rates studied. In order to increase sample throughput the maximum flow rate attainable with the pump tubing used of $6 \mathrm{ml} \mathrm{min}^{-1}$ was used.

The flow rate at which the liquid waste was removed from the gas-liquid separator was varied from 3 to $8 \mathrm{ml} \mathrm{min}^{-1}$ and it was found that the flow rate did not affect the analytical response. However, if the flow rate was too low, for example less than $4 \mathrm{ml} \mathrm{min}^{-1}$, such that the rate of production of spent liquid was not matched by the rate of liquid removal from the gas-liquid separator then flooding the manifold with the waste liquid was possible. A flow rate of $8 \mathrm{ml} \mathrm{min}^{-1}$ was therefore chosen for further experiments.

\section{Investigation of the Effect of Rf Power on the Sensitivity}

The response of the spectrometric signals from elements introduced into an ICP is dependent on the power density of the plasma. The effect of the rf power on the response for mercury was studied by noting the analytical sensitivity as a function of varying rf power with all other parameters held constant. The results, shown in Fig. 5, indicate that at an rf power of $1000 \mathrm{~W}$, the maximum sensitivity for mercury was obtained. The purge gas flow rate was re-optimized to obtain the maximum signal intensity. Rf power and the purge gas flow rate operate as a paired set of variables since if one is changed, the other must be changed to re-optimize the signal intensity. ${ }^{20}$

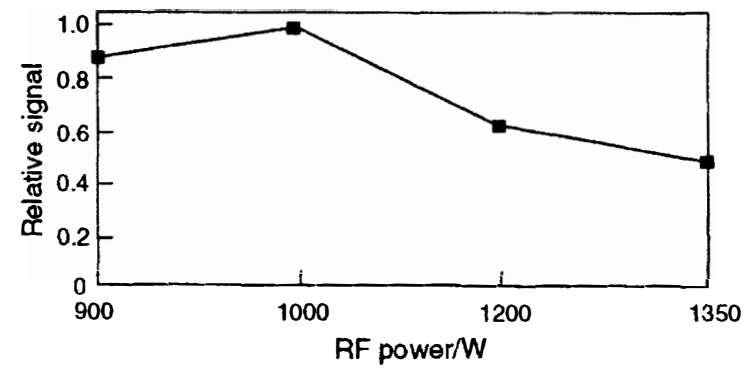

Fig. 5 Analytical sensitivity of mercury as a function of rf power 


\section{Precision}

This study was carried out with a view to addressing the analytical questions, namely, stability during operation and drift for the mercury response caused by carryover and contamination for extended periods of continuous operation. These are important considerations since there is no suitable element to use as an internal standard in this method. The precision was studied using a $1 \mu \mathrm{g}^{-1}$ standard of mercury and $1.1 \% \mathrm{~m} / \mathrm{v}$ tin(II) chloride. The precision, calculated by the relative standard deviation, was $0.9 \%$ for 21 determinations. Each determination was the result of three replicate measurements.

\section{Investigation of the Analytical Response as a Function of Mass of Analyte Preconcentrated}

One of the advantages of using time-based injection is that it allows different amounts of sample to be chosen without manifold changes. Sensitivities of upwards of 1 billion counts $\mathrm{s}^{-1}$ per $\mathrm{mg} \mathrm{l}^{-1}$ of mercury are obtainable with this combination, which is an order of magnitude better than is obtained for mercury by conventional cold vapour generation techniques. ${ }^{12}$ One of the analytical implications of the linear relationship between mass of sample and the sensitivity is that it is possible to generate a calibration plot using a single standard.

\section{Detection Limits}

The detection limits were calculated by the three standard deviation criterion using $18 \mathrm{M} \Omega$ de-ionized water as blank. Fig. 6 shows a plot of detection limit as a function of volume of sample injected. An improvement in the detection limit was observed as the sample volume was increased. A detection limit of $200 \mathrm{pg}^{-1}$ was measured for a sample volume of $25 \mathrm{ml}$. It should be pointed out, however, that the signal-to-noise ratio also increases as the volume of sample injected is increased and this manifests itself in the marginal gain in detection limit as the sample volume is increased between 10 and $25 \mathrm{ml}$. It is often suggested in publications dealing with the use of solidphase extractants that the detection limit of the resulting procedure can be improved to any desired value by the simple expedient increase of the sample volume. In general, for a procedure that requires the use of materials in addition to the sample, this is not true. Eventually, as the sample volume is increased, the contamination of reagents, carrier streams, etc., with the analyte species becomes the dominant source of measurement uncertainty and thus detection limits are set not by instrumental factors but by reagent purity. To a large extent the purity of reagents determines the detection limits achievable with FI-amalgamation-ICP-MS.

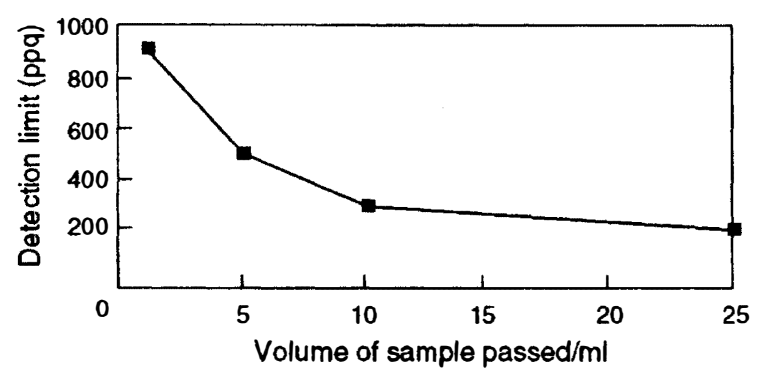

Fig. 6 Detection limit as a function of sample volume injected

Table 4 Spike recovery results for amalgamation-ICP-MS

Sample

NRCC NASS-4 Sea-water

NRCC NASS-2 Sea-water

NRCC CASS-4 Sea-water

NRCC SLRS-1 Riverine Water

NIST SRM 1643c Water

Table 5 Quantitative analysis of NIST SRM 1641c using vapour generation amalgamation-ICP-MS

\begin{tabular}{lcc}
\hline Analyte & Certified value $/ \mu \mathrm{g} 1^{-1}$ & Found $/ \mu \mathrm{g} 1^{-1 *}$ \\
NIST SRM 1641c & $0.65 \pm 0.10$ & $0.65 \pm 0.12$ \\
\hline
\end{tabular}

$* \pm$ term is the $95 \%$ confidence interval for five replicate measurements.

\section{Application to Fresh and Saline Water}

Spike recovery studies

Mercury was spiked into Water, NIST SRM 1643c, Riverine Water, NRCC SLRS-1 and Sea-waters (Open-Ocean and Coastal) NRCC CASS-2, NRCC NASS-2 and NRCC NASS-4 at known levels and then determined by the proposed method. Unspiked samples of the SRM or CRM were also analysed; the recoveries were calculated from the difference between the concentration of mercury in the spiked and unspiked samples. The results for the spike recovery are shown in Table 4 . The recoveries indicate that the method is not subject to significant matrix interferences which would have resulted in either enhancement or suppression of analyte signal for the sample matrices studied. Although none of the SRMs or CRMs used was certified for mercury, all of them, except for SLRS-1, showed mercury concentrations of about $0.9 \mu \mathrm{g}^{-1}$ when analysed using the proposed method.

\section{Quantitative analysis of NIST SRM 1641c}

The result of the determination of mercury is summarized in Table 5. The NIST SRM 1641c, Mercury in Water, was diluted to a concentration of about $0.6 \mu \mathrm{g}^{-1}$ and analysed. The results obtained using external calibration show good agreement between the mercury concentration found using the proposed method and the certified value. The uncertainty reported is the $95 \%$ confidence interval for five replicate measurements.

\section{CONCLUSION}

The combination of chemical vapour generation implemented by an automated flow injection procedure, in combination with ICP-MS, can be used for the accurate determination of low concentrations of mercury in river, saline and potable water.

\section{REFERENCES}

1 Ward, N. I., in Applications of Inductively Coupled Plasma Mass Spectrometry, ed. Date, A. R., and Gray, A. L., Blackie, London, 1989, pp. 189-219.

2 Houk, R. S., Fassel, V. A., Flesch, G. D., Svec, H. J., Gray, A. L., and Taylor, C. E., Anal. Chem., 1980, 52, 2283.

3 Boomer, D. W., and Powell, M. J., Anal. Chem., 1987, 59, 2810.

4 Fang, Z-L., Xu, S-K., Wang, X., and Zhang, S-C., Anal. Chim. Acta, 1986, 179, 325.

5 Fang, Z-L., Xu, S-K., Wang, X., Zhang, S-C., Guo, L., and Sun, L-J., Anal. Chim. Acta, 1988, 214, 41.

6 de Andrade, J. C., Pasquini, C., Baccan, W., and van Loon, J. C., Spectrochim. Acta, Part B, 1983, 38, 1329.

7 Guo, T. Z., and Baasner, J., Anal. Chim. Acta, 1993, 278, 189. 
8 Guo, T. Z., and Baasner, J., Talanta, 1993, 40, 1927.

9 Pasquini, C., Jardim, W. F., and de Faria, L. C., J. Autom. Chem. 1988, 10, 188.

10 Marshall, G. D., and van Staden, J. F., J. Anal. At. Spectrom., 1990, 5, 675 .

11 Rudner, P. C., de Torres, A. G., and Pavon, J. M. C., J. Anal. At. Spectrom., 1993, 8, 705.

12 Stroh, A., and Völlkopf, U., J. Anal. At. Spectrom., 1993, 8, 35.

13 Haraldsson, C., Westerlund, S., and Öhman, P., Anal. Chim. Acta, 1989, 221, 77.

14 Plantz, M. R., Fritz, J. S., Smith, F. G., and Houk, R. S., Anal. Chem., 1989, 61, 149.

15 Beauchemin, D., McLaren, J. W., Willie, S. N., and Berman, S. S., Anal. Chem., 1988, 60, 687.
16 Hanna, C. P., Haigh, P. E., Tyson, J. F., and McIntosh, S., J. Anal. At. Spectrom., 1993, 8, 585.

17 Douglas, D. J., and French, J. B., Spectrochim. Acta, Part B, 1986, 41, 197.

18 Erler, W., Perkin-Elmer Technical Summary TSAA-9A.

19 McIntosh, S., Perkin-Elmer Technical Summary TSAA-10B.

20 Zhu, G., and Browner, R. F., Appl. Spectrosc., 1987, 41, 349.

Paper 5/05406K

Received August 11, 1995 Accepted October 17, 1995 